\title{
Avaliação da Maturidade em Colaboração de Equipes Ágeis de Desenvolvimento de Software
}

\author{
Carlos Eduardo Stefani ${ }^{1}$, Marcelo Duduchi ${ }^{1}$ \\ ${ }^{1}$ Centro Paula Souza (CPS) \\ Rua dos Bandeirantes, 169 - Bom Retiro, São Paulo - SP - Brasil \\ cafani@gmail.com, mduduchi@gmail.com
}

\begin{abstract}
This paper describes an ongoing research that seeks to elaborate a process for assessing the collaborative maturity of teams that develop software using agile methods. As preliminary results, it presents a literature review and the result of a Survey on Encouraging and Using Collaboration in Agile Methods that drew 271 respondents.

Resumo. Este artigo descreve uma pesquisa em andamento que busca elaborar um processo para avaliar a maturidade em colaboração de equipes que desenvolvem softwares utilizando de métodos ágeis. Como resultados preliminares, apresenta uma revisão da bibliografia e o resultado de uma Survey sobre incentivo e uso da colaboração em métodos ágeis que obteve 271 respondentes.
\end{abstract}

\section{Contexto do trabalho}

Os processos de desenvolvimento de software passaram por grandes transformações nas últimas décadas, necessitando maior interação entre os envolvidos. Cada vez são mais utilizados modelos baseados em processos mais flexíveis e incrementais, como os métodos ágeis. Estes buscam a entrega contínua de valor de partes do software, buscando melhoria contínua tanto do produto de software quanto do seu processo de desenvolvimento (PRESSMAN, 2011). Estas transformações vêm ao encontro de um mercado em constante mudança, em que é necessário adaptar-se continuamente e agir de modo eficiente para que o resultado produzido pelo software seja efetivo para os negócios das organizações (GOLDMAN, NAGEL e PREISS, 1994).

Parte das contribuições dos métodos ágeis é o incentivo à comunicação e colaboração. Quanto melhor for a colaboração entre os membros dos times de desenvolvimento, patrocinadores, clientes e os demais envolvidos, mais bem direcionados serão os esforços para a produção do software, mais fluida será a resposta contínua às mudanças, maior será a capacidade de produzir software alinhado às necessidades de negócio e de inovação e maior será a qualidade do software aferida sob a ótica do usuário (BECK et al., 2001; HIGHSMITH E COCKBURN，2001; COCKBURN，2002; JACOBSON，2002; PRESSMAN, 2011).

Além disso, segundo Jacobson (2002), uma equipe ágil reconhece que as capacidades individuais somadas à capacidade de colaborar são fatores principais de sucesso de um projeto. Pressman (2011) ressalta que agilidade vai além de resposta a mudanças, "incentiva a estruturação e as atitudes em equipe que tornam a comunicação mais fácil (entre membros da equipe, entre o pessoal ligado à tecnologia e o pessoal da área comercial, entre os engenheiros de software e seus gerentes)" (PRESSMAN, 2011, p. 83). 


\section{Problema a ser investigado}

Atualmente, as organizações enfrentam problemas de tamanha complexidade, que nenhum indivíduo possui conhecimento, influência e recursos para resolvê-los sozinho. Neste cenário, a colaboração se tornou ubíqua na vida das organizações (VREEDE, MASSEY e BRIGGS, 2009).

As organizações ainda possuem dificuldade em integrar práticas, técnicas e características de colaboração aos processos ágeis de desenvolvimento de software que permitam cooperação, fluidez da comunicação e flexibilização na coordenação capazes de melhorar o resultado dos projetos de software.

Neste contexto, este estudo guia-se pela seguinte questão de pesquisa: Quais elementos devem compor um processo de avaliação que permita aferir o nível de maturidade em colaboração de equipes inseridas no processo de desenvolvimento de software?

O objetivo geral deste estudo é desenvolver um processo que permita aferir o nível de maturidade em colaboração de equipes de desenvolvimento de software nas empresas, de forma que possa ser utilizado como guia para diagnosticar e evoluir a colaboração nos processos de desenvolvimento de software.

Como objetivos específicos, busca:

- Encontrar fatores de colaboração pertinentes às equipes de desenvolvimento de software em métodos ágeis;

- Propor uma classificação dos fatores em níveis de maturidade de colaboração utilizando como base o ColabMM (MAGDALENO, 2016);

- Definir um processo de avaliação da colaboração em equipes de desenvolvimento de software;

- Avaliar a situação de equipes quanto à utilização de colaboração.

\section{Fundamentação}

Nesta seção são apresentadas definições que fundamentam a pesquisa.

\subsection{Colaboração em Métodos Ágeis de Desenvolvimento de Software}

De acordo com Dingsøyr at al. (2012), os métodos ágeis de desenvolvimento de software surgiram a partir de experiências pessoais e conhecimento coletivo da comunidade envolvida no desenvolvimento de software. Nos métodos ágeis são priorizados "indivíduos e interações acima de processos e ferramentas, software operacional acima de documentação completa, colaboração dos clientes acima de negociação contratual e respostas a mudanças acima de seguir um plano" (BECK et al., 2001). Colaboração com o cliente significa que todos os envolvidos - patrocinadores, clientes, usuários e desenvolvedores - estão no mesmo time (HIGHSMITH e COCKBURN, 2001).

O manifesto ágil (BECK et al., 2001), um marco para a adoção dos métodos ágeis, elegeu 12 princípios que devem ser adotados ao utilizar métodos ágeis. Dos 12 princípios, 6 deles tratam de colaboração de forma direta ou indireta (profissionais técnicos e de negócio trabalhando em conjunto diariamente; indivíduos motivados e compartilhando ambiente propício; conversa cara a cara; ambiente sustentável com passos constantes; equipes auto organizáveis e; reflexões em equipe), tendo relação direta aos objetivos desta pesquisa. 


\subsection{Teorias e Modelos de Colaboração}

Segundo Vreede e Briggs (2005), colaboração significa unir esforços para atingir um objetivo do grupo. Segundo os autores, isto não significa que os membros do grupo concordam ou se sintam felizes com o objetivo, somente requer que, por qualquer razão, trabalhem para a sua obtenção.

Para o Modelo 3C, colaboração é a combinação entre comunicação, coordenação e cooperação. Comunicação representa a troca de informações e mensagens entre as pessoas, coordenação é o gerenciamento das pessoas, atividades e recursos e cooperação é o compartilhamento do espaço de trabalho (FUKS et al., 2005). Nele, a comunicação é feita de e para todos os integrantes do grupo, sendo voltada para a ação. As pessoas se comunicam ao mesmo tempo em que tomam decisões. Os membros do grupo se auto coordenam ao organizar suas atividades e lidar com conflitos de forma a otimizar a atuação do grupo, utilizando comunicação. Durante a cooperação normalmente surgem questões que exigem comunicação e coordenação. As três atividades, portanto, se complementam e auto relacionam (PIMENTEL e FUKS, 2012).

De acordo com Briggs et al. (2006), padrões de colaboração são as atividades e a forma de executá-las que permitem que o grupo colabore para atingir seu objetivo. Podem ser distribuídos em seis grupos: geração, redução, esclarecimento, organização, avaliação e construção de consenso. Já segundo Vreede, Massey e Briggs (2009), em um ponto de vista mais holístico, com detalhes das práticas de trabalho, tecnologias e guias para padrões de colaboração, têm-se a Engenharia da Colaboração. Ela é especialmente útil quando trata de tarefas colaborativas de missão crítica. No desenvolvimento de software, destacam: negociação e especificação de requisitos, teste de usabilidade, inspeção dos requisitos e inspeção de código.

Existem alguns modelos propostos de maturidade em colaboração. Entre eles, destacamse: The Fast Reactive Extended Enterprise-Capability Assessment Framework (FREECAF); Collaboration Maturity Grid (CMG); SAP AG; Col-MM (BOUGHZALA e VREEDE, 2012) e ColabMM (MAGDALENO, 2016). Para este estudo optou-se pela utilização do modelo ColabMM como referência, pois é voltado a processos de negócios.

\subsection{Trabalhos correlatos}

Foram encontrados 466 trabalhos que tangenciam o tema desta pesquisa na base Scopus até 2018 (Figura 1). Observa-se ascendência de publicações, com tendência de crescimento.

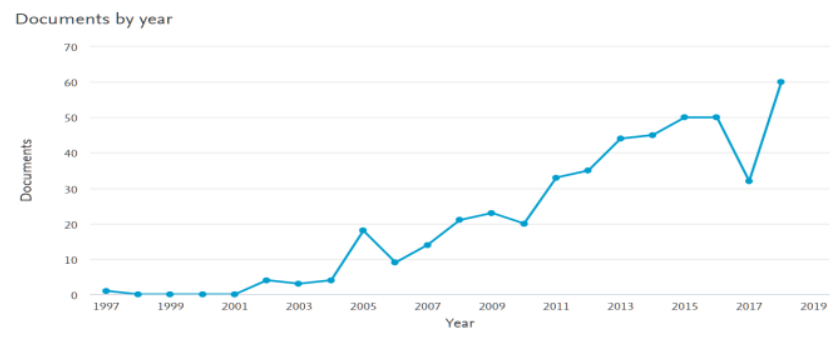

Figura 1. Documentos publicados por ano na base Scopus

Selecionando, entre os documentos encontrados, os que tratam de maturidade, temos no Quadro 1 os cinco documentos mais citados. Observa-se que os artigos tratam de ambiente físico, melhoria do processo de software, ensino de colaboração em métodos 
ágeis, maturidade do grupo e otimização da colaboração.

\section{Quadro 1. Documentos sobre maturidade em colaboração e métodos ágeis}

\begin{tabular}{|l|c|l|c|}
\hline Autores & Ano & Título & Citações \\
\hline Mishra, D., Mishra, A., Ostrovska, S. & 2012 & $\begin{array}{l}\text { Impact of physical ambiance on communication, collaboration and } \\
\text { coordination in agile software development: An empirical } \\
\text { evaluation }\end{array}$ & 26 \\
\hline Salo, O., Abrahamsson, P. & 2005 & $\begin{array}{l}\text { Integrating agile software development and software process } \\
\text { improvement: A longitudinal case study }\end{array}$ & 24 \\
\hline $\begin{array}{l}\text { Fontana, R.M., Fontana, I.M., Da Rosa } \\
\text { Garbuio, P.A., Reinehr, S., Malucelli, A. }\end{array}$ & 2014 & $\begin{array}{l}\text { Processes versus people: How should agile software development } \\
\text { maturity be defined? }\end{array}$ & 23 \\
\hline Meier, A., Kropp, M., Perellano, G. & 2016 & $\begin{array}{l}\text { Experience report of teaching agile collaboration and values: Agile } \\
\text { software development in large student teams }\end{array}$ & 7 \\
\hline Suscheck, C.A., Ford, R. & 2008 & $\begin{array}{l}\text { Jazz improvisation as a learning metaphor for the scrum software } \\
\text { development methodology }\end{array}$ & 7 \\
\hline
\end{tabular}

Para condução desta pesquisa, considerando o desenvolvimento de software como um processo organizacional, planeja-se utilizar de base predecessora o trabalho de Magdaleno (2016), uma vez que estabelece níveis de maturidade em colaboração para processos organizacionais e suas práticas requeridas (Figura 2).

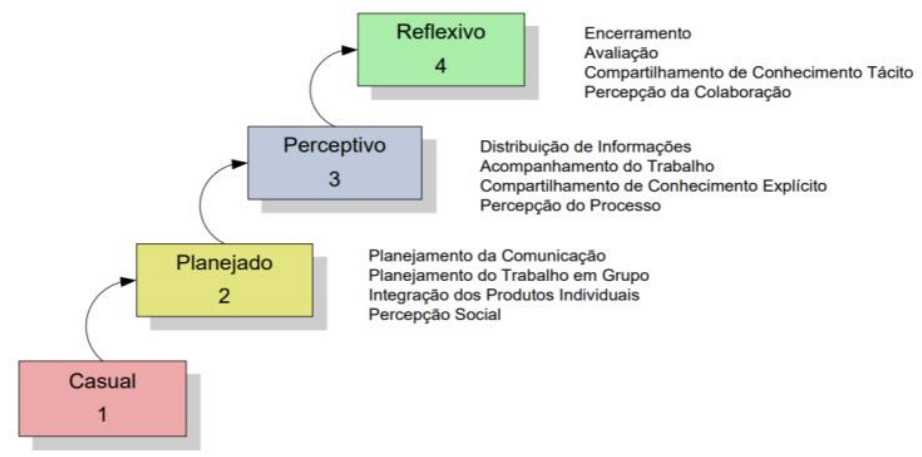

Figura 2: Práticas de colaboração em níveis de maturidade. Fonte: Magdaleno (2016)

Apesar de existirem pesquisas recentes que tangenciam a proposta desta, como em Fontana et al. (2014), Gren et al. (2015) e Magdaleno et al. (2015), são escassos os estudos que tratam de colaboração no próprio desenvolvimento de software (NEIVA et al., 2016). Um importante desafio para esta pesquisa é identificar os fatores e particularidades de colaboração do desenvolvimento ágil de software e definir as posições destes fatores nos níveis de maturidade em colaboração propostos por Magdaleno (2016).

\section{Procedimentos da pesquisa e resultados preliminares}

A Figura 3 apresenta o plano completo da pesquisa. O item 1 (Survey incentivo e uso de colaboração na produção de software) já foi realizado e o item 2 está em andamento. Os resultados obtidos e planos para os demais itens são apresentados nas seções a seguir.

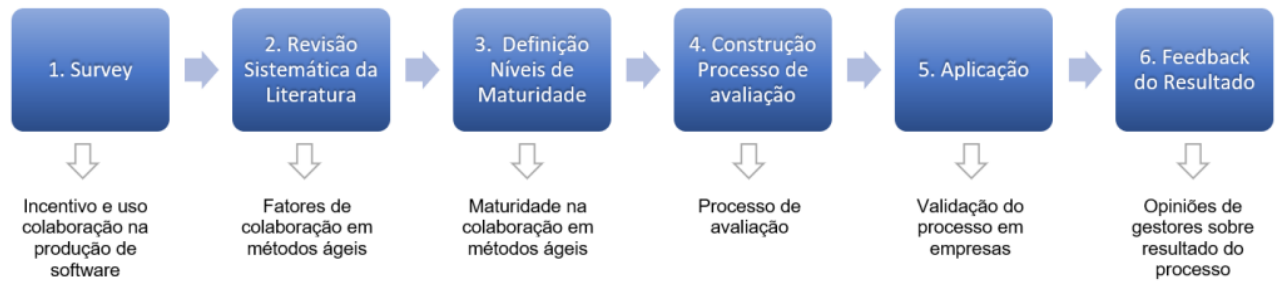

Figura 3. Procedimentos da pesquisa

\subsection{Survey sobre Incentivo e Uso de Colaboração na Produção de Software}

O estudo buscou verificar, em processos de desenvolvimento de software, quanto as organizações incentivam a colaboração e quanto os profissionais envolvidos fazem uso 
de técnicas de colaboração. Foi realizado levantamento por questionário com amostra de 271 respondentes, variada quanto ao tipo, porte e natureza da organização e quanto ao modelo de processo de software adotado e tipo de software desenvolvido. O resultado da pesquisa quanto ao incentivo e ao uso da colaboração foi bastante disperso na escala Likert de 1 a 5 utilizada, indicando pouco consenso tanto por parte das organizações em incentivar a colaboração quanto por parte dos profissionais em utilizá-la (Figura 4).
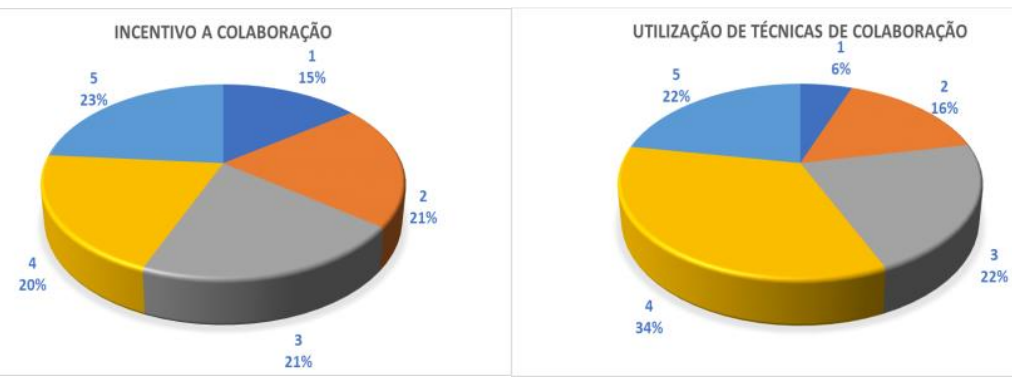

Figura 4. Utilização de técnicas de colaboração no desenvolvimento de software

Entretanto, como mostrado nos gráficos da Figura 5, observou-se que quanto maior a utilização de métodos ágeis, maior o incentivo e uso da colaboração, corroborando com o referencial teórico apresentado.

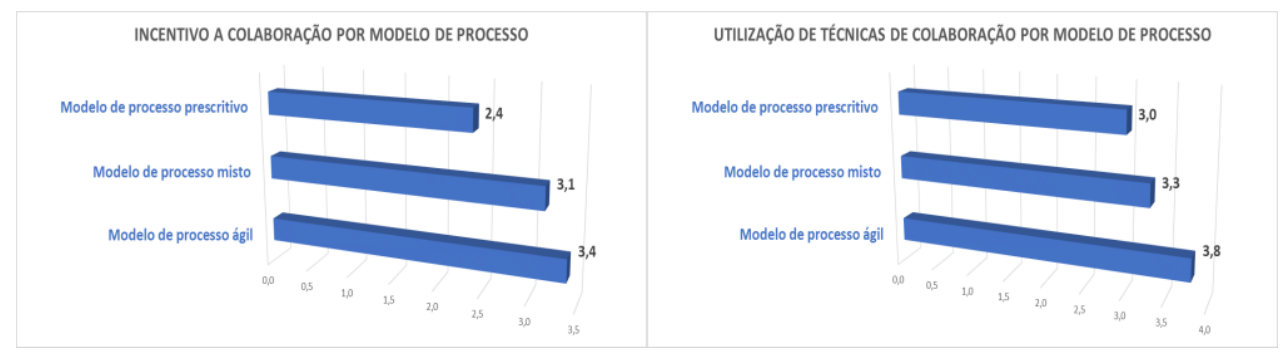

Figura 5. Incentivo e uso de colaboração por modelo de processo de software

Na Tabela 1 é possível observar que a maior correlação encontrada ocorre entre o incentivo da colaboração por parte das organizações e uso de colaboração por parte dos respondentes $(0,530)$. Foram também encontradas leves correlações do modelo de processo com o uso de colaboração $(0,259)$ e incentivo à colaboração $(0,199)$.

Tabela 1. Correlações de postos de Spearman com colaboração

\begin{tabular}{|l|r|r|}
\hline & Incentivo à colaboração & Utilização da colaboração \\
\hline Tipo de Organização &, $153^{*}$ &, $235^{* *}$ \\
\hline Porte da organização & 0,072 & $-0,044$ \\
\hline Natureza da organização &, $122^{*}$ &, $218^{* *}$ \\
\hline Modelo de Processo &, $199^{* *}$ &, $259^{* *}$ \\
\hline Incentivo à colaboração & 1,000 &, $530^{* *}$ \\
\hline Utilização da colaboração &, $530^{* *}$ & 1,000 \\
\hline
\end{tabular}

\subsection{Continuidade da pesquisa}

Pretende-se, a seguir, relacionar os elementos de colaboração presentes em métodos ágeis de desenvolvimento de software encontrados na literatura com os níveis de colaboração propostos por Magdaleno (2016), o que permitirá, no processo de avaliação, determinar o nível em que se encontram, utilizando como referência a utilização dos elementos. $\mathrm{Na}$ sequência, pretende-se construir o processo de avaliação que com perguntas permitam identificar nível de colaboração em equipes de desenvolvimento de software que utilizam métodos ágeis e aplicá-lo para verificar sua efetividade. 


\section{Referências}

BECK et al. Manifesto para desenvolvimento ágil de software. 2001. Disponível em: $<$ http://agilemanifesto.org>, acesso em 09/06/2018.

BOUGHZALA, I E VREEDE, G. A Collaboration Maturity Model: Development and Exploratory Application. 45th Hawaii International Conference on System Sciences. IEEE. Maui, 2012.

COCKBURN, A. Agile Software development: the cooperative game. 2nd edition. Addison-Wesley. 2006.

DINGSØYR, T., NERUR, S. P., BALIJEPALLY, V.; MOE, N. B. A decade of agile methodologies: Towards explaining agile software development. Journal of Systems and Software, 85. 2012.

GREN et al. Group maturity and agility, are they connected? - A survey study. 41st Euromicro Conference on Software Engineering and Advanced Applications. Portugal, 2015.

FONTANA, et al. Processes versus people: How should agile software development maturity be defined? Journal of Systems and Software. Volume 97. 2014

FUKS, H. et al. Applying the $3 \mathrm{C}$ model to groupware development. International Journal of Cooperative Information Systems. Vol. 14. 2005.

GOLDMAN, S.L; NAGEL, R. N.; PREISS, K. Agile Competitors and Virtual Organizations: Strategies for Enriching the Customer. John Wiley Trade. 1994.

HIGHSMITH, J. E COCKBURN, A. Agile software development: the business of innovation. Computer, vol. 34, no. 9. IEEE Computer Society. Setembro, 2001.

JACOBSON, I. A Resounding Yes to Agile Process - But Also More. Cutter IT Journal, v. 15, n. 1, p. 18-24. 2002.

MAGDALENO et al. Collaboration optimization in software process composition. Journal of Systems and Software. Volume 103. 2015.

MAGDALENO, A. M. Explicitando a Colaboração em Organizações através da Modelagem de Processos de Negócio. Universidade Federal do Rio de Janeiro. Rio de Janeiro, 2016.

NEIVA et al. Interoperability Requirement to Enhance Collaboration in Software Product Lines: A Systematic Mapping. 13 Simpósio Brasileiro de Sistemas Colaborativos. SBC. Rio Grande do Sul, 2016.

PIMENTEL, M.; FUKS, H. Sistemas Colaborativos. Elsevier. Rio de Janeiro, 2011.

PRESSMAN, R. S. Engenharia de software: uma abordagem profissional. $7^{\mathrm{a}}$ edição. Porto Alegre: AMGH, 2011.

VREEDE, G.J. DE; BRIGGS, R.O. Collaboration Engineering: Designing Repeatable Processes for High-Value Collaborative Tasks. Proceedings of the Hawaii International Conference on System Science. IEEE Computer Society Press. Los Alamitos, 2005.

VREEDE, G.; MASSEY, A. P.; BRIGGS, R. O. Collaboration Engineering: Foundations and Opportunities. Journal of the Association of Information Systems. v. 10. 2009. 